\title{
3 Research Square \\ Loss to follow-up and associated maternal factors among HIV-exposed infants at the Mbarara Regional Referral Hospital, Uganda: A retrospective study
}

\section{Rogers Ankunda}

Mbarara University of Science and Technology

Samuel Nambile Cumber

University of Pretoria

Catherine Atuhaire ( $\nabla$ catuhaire@must.ac.ug)

Mbarara University of Science and Technology https://orcid.org/0000-0002-6028-7491

Kabanda Taseera

Mbarara University of Science and Technology

Claude Ngwayu Nkfusai

University of Buea Faculty of Health Sciences

Frankline Sevidzem Wirsiy

University of Buea Faculty of Health Sciences

Turyakira Eleanor

Mbarara University of Science and Technology

Research article

Keywords: HIV-exposed infants, Loss to follow up, Post-natal mother to child transmission

Posted Date: March 10th, 2020

DOI: https://doi.org/10.21203/rs.3.rs-16526/v1

License: (9) (i) This work is licensed under a Creative Commons Attribution 4.0 International License.

Read Full License

Version of Record: A version of this preprint was published at BMC Infectious Diseases on March 19th, 2020. See the published version at https://doi.org/10.1186/s12879-020-04964-1. 


\section{Abstract}

\section{Background}

Loss to follow-up (LTFU) deprives HIV-exposed infants the lifesaving care required and results in exposing HIV free infants to virus requisition risk. We aimed to determine the rate of LTFU, postnatal mother-to-child HIV-transmission and to identify maternal factors associated with LTFU among HIVexposed infants enrolled at Mbarara Regional Referral Hospital PMTCT clinic.

\section{Methods}

Study participants were infants born to HIV-positive mothers enrolled in the PMTCT clinic for HIV care at Mbarara Regional Referral Hospital. While access database in the Early Infant Diagnosis (EID) clinic provided data on infants, the open medical record system database at the ISS clinic provided that for mothers. Infants were classified as LTFU if they had not complete their follow-up schedule by 18 months of age. At 18 months, an infant is expected to receive a rapid diagnostic test before being discharged from the PMTCT clinic. Postnatal MTCT of HIV was calculated as a proportion of infants followed and tested from birth to 18 months of age. Logistic regression was used to determine possible associations between mothers' characteristics and LTFU. In-depth interviews of mothers of LTFU infants and health workers who attend to the HIV-exposed infants were carried out to identify factors not captured in the electronic database.

Results

Out of 1624 infants enrolled at the clinic, 533 (33\%) were dropped for lack of mother's clinic identification number, 18 (1.1\%) were either dead or transferred out. Out of 1073 infants analysed, 515 (48\%) were LTFU while out of the 558 who completed their follow-up schedule, $20(3.6 \%)$ tested positive for HIV. Young age of mother, far distance to hospital and non-use of family planning were identified as outstanding factors responsible for LTFU. In addition, in-depth interviews revealed facility-level factors such as "waiting time".

\section{Conclusion}

This study has revealed a high rate of LTFU among HIV-exposed infants enrolled at Mbarara Regional Referral hospital PMTCT clinic. Young maternal age, long distance to health facility and failure to use family planning were significantly associated with LTFU. Incorporating family planning services in the ART and PMTCT clinics could reduce LTFU of these infants.

\section{Background}

The impact of HIV on children cannot be over emphasised. The 2013 global statistics revealed that 3.2 million children under 15 years old, $9.1 \%$ of the global population, were living with the virus [1]. In subSaharan Africa lives $91 \%$ of children with the virus with vertical transmission a leading cause of infant 
infection[2], $6 \%$ of HIV infected children are in Asia and the Pacific and the remaining $3 \%$ live in other parts of the world[1]. By a 2013 appraisal, 1.3 million women living with HIV were delivered of their babies without any change from 2009 [1]. A significant drop of mother-to-child transmission rate of HIV from $25.8 \%$ in 2009 to $16 \%(13-18 \%)$ in 2013 was recorded [1]. Annually, nearly 1.4 million HIV-1 positive women conceive, majority of whom are from sub-Saharan Africa [2]. The proportion of women living with HIV-1 among antenatal clients in sub-Saharan Africa ranges from $5 \%$ to as high as $30 \%$.

In Uganda, there are 1.2 million HIV infected people with $57 \%$ made of females and $13 \%$ children while new infections were estimated at 124,000 [3]. In 2012, the prevalence of HIV among adults was at $7.2 \%$ with 780,000 women and 190,000 children living with HIV respectively. In Uganda, 160000 children became infected with HIV and 328 HIV related deaths were reported every day [4]. The 2010 PMTCT Annual Report projected that in 2010, 1,550,000 pregnancies occurred increased to 1,600,000 in 2011 in Uganda. This could be translated into 100,750 and 104,000 pregnant women living with HIV in 2010 and 2011 respectively [3].

HIV - 1 can be transmitted from mother-to-child during pregnancy, labor and delivery and after birth through breastfeeding [5]. The risks of viral transmission during pregnancy ranges from 5-10\%, during labor and delivery from $10-20 \%$, and during mixed infant feeding from $10-20 \%$. In the absence of any intervention to prevent mother- to-child transmission (MTCT), it is estimated that the viral transmission risk will rise rapidly from $15-45 \%$. Therefore, with effective interventions, this risk can be reduced to levels below $5 \%$ [6]. Over the years, there have been milestone research and developments in knowledge and interventions that can mitigate and save lives of infants exposed to HIV Virus.

The World Health Organization highly recommend for HIV-exposed infants to be tested using HIV-1 DNA PCR test. Yet, in 2013 , only $42 \%$ of infants born to mothers living with HIV in low and middle-income countries received this test within 2 months as recommended. In Uganda, only $36 \%$ of HIV-exposed infants were tested [1] mainly because they were not brought for follow-up care in Early Infant HIV Diagnosis (EID) clinics.

The failure of HIV exposed infants to return for follow-up care puts those born free of HIV (whose $1^{\text {st }}$ PCR was negative) at risk of acquiring HIV and deprives those who have already acquired the virus the necessary care they need to stay alive.

In more than a week period, over $48 \%$ of infants receiving EID services at Mbarara Regional Referral Hospital miss their appointments. This study sought to determine the proportion of loss to follow-up and identify maternal factors associated with loss to follow-up among HIV exposed infants enrolled at Mbarara Hospital PMTCT clinic (unpublished EID registers, 2012).

\section{Methods}

Study design: This was a mixed study design comprising a descriptive retrospective registry-based cohort study and In-depth Interviews. The retrospective study was carried out on infants born to HIV positive 
mothers attending the PMTCT/EID clinic at Mbarara Hospital Immune Suppression Syndrome (ISS) clinic. In addition, qualitative interviews of mothers whose babies were lost to follow-up and health workers in the EID/PMTCT clinic were carried out.

Study setting: The study was conducted at the ISS clinic of Mbarara Regional Referral Hospital (MRRH) in Mbarara district. Mbarara district is found in the south-western part of Uganda, and is located $270 \mathrm{kms}$ from Kampala city. At this hospital like in all maternal child health $(\mathrm{MCH})$ facilities, all pregnant women attending antenatal care (ANC) clinic receiving counselling, are tested for HIV and are recorded in PMTCT or ANC registers. All HIV positive women are recorded in PMTCT care registers and instantly placed on lifelong ART treatment regardless of CD4 count or gestation period. They are started on ART combination of TDF, 3TC and EFV. Infants born to mothers infected by HIV are documented in the EID register after birth, and followed up till they are 18 months old and within this time, mothers receive counselling on infant feeding along with ARV prophylaxis for PMTCT of HIV. To this effect, infants whose mothers are placed on lifelong ART are given once daily NVP from birth to 6 weeks of age regardless of whether they are exclusively breastfed or given replacement feeding and thereafter receive cotrimoxazole prophylaxis. According to the Uganda HIV infant testing algorithm, an HIV DNA PCR test is carried out at $\leq 6$ weeks of age and cotrimoxazole is then started. In the case where the $1^{\text {st }}$ DNA PCR is negative and the child has been breastfeeding, the $2^{\text {nd }}$ DNA PCR test is carried out 6 weeks after breastfeeding has stopped. When a $2^{\text {nd }}$ PCR turns out to be negative, a rapid HIV test is conducted at 18 months old prior exit of care by child. On the other hand, if the $1^{\text {st }}$ or $2^{\text {nd }}$ PCR is positive, the infant is referred for ART initiation. By June, 2014, the EID/PMTCT clinic at Mbarara Hospital ISS clinic had 3120 infant's cumulative enrolment since its inception in 2005, and by January 2013, 2072 infants enrolled.

Study population: The study was delimited to HIV exposed infants who enrolled for care at the Mbarara Hospital PMTCT/EID clinic between January 2010 and January 2013 and whose mothers received care from Mbarara Regional Referral Hospital ISS clinic during the same period. Infants whose mothers' lacked clinic identification numbers were deemed ineligible.

Sample size estimation: All HIV-exposed infants who had been enrolled from January 2010 to January 2013 and followed up for 18 months and whose mothers received care from ISS clinic were analysed. A sample size of 772 mother-infant pairs was estimated based on assumptions of $80 \%$ power; odds ratio for loss to follow up of 2.2 [7] and level of significance of 0.05 (two-sided). This estimate was reached at using a method in observational epidemiology for calculating sample size for unmatched cross-sectional studies, cohort studies and randomized clinical trials [7][8].

Quantitative data collection: Data on HIV-exposed infants were obtained from the Access database system for the PMTCT/EID clinic whereas, data on mothers were obtained from the electronic Open Medical Record System (Open MRS), the database for the ISS clinic. Data of eligible mother-infant pairs were identified by unique client numbers in the electronic databases and the dataset converted into STATA format (Stata Corporation Inc.). The main outcome variable was loss to follow-up among HIVexposed infants. An infant was classified loss-to follow-up if he/she did not complete follow up to the 
point of being discharged and was not declared deceased. Data on independent variables were extracted as recorded in databases and later categorised where necessary as described by Kabakyenga and colleagues(1). For example, age was categorised and coded as 0 "18-23", 1 "24-29" and 2 ">30 years".

Qualitative data collection: Fourteen interviews were conducted, ten of them with selected HIV positive mothers whose exposed infants were lost to follow up and four with health workers who attend to these infants. The purpose of the qualitative interviews was to capture factors associated with LTFU that could have been missed out in the electronic database of the ISS clinic such as facility-level client experiences and health worker behaviours. Tracing of mothers of lost babies was done by phone calls after their information had been generated from the data set. Only mothers who had left their phone contacts with the clinic were contacted. Interviews were audio recorded, transcribed word verbatim and translated approximately from the local dialect to English language for analysis.

Data analysis: Quantitative data analysis was done using Stata version 11.0 (Stata Corp, College Station, TX, USA). Frequency counts and percentages were obtained to describe socio-demographics and other categorical variables. The median and inter-quartile ranges were used to describe quantitative variables with skewed distributions such as CD4 count. In the bivariate analysis, independent variables were cross tabulated with the outcome variable to determine possible associations. Odds Ratios and their $95 \%$ confidence intervals were calculated. Independent variables with $p$-value $\leq 0.2$ in the bivariate analysis were entered into a multivariable logistic regression model to adjust for confounding. Statistical significance of variables in the final model was assessed based on a p-value threshold of $\leq 0.05$. In analysing the qualitative data, English transcripts were read in between the lines to identify codes and themes. Identification of codes was guided by the conceptual framework and study objectives with room for emergent themes from the data. Coding and analysis were done manually using a cut and paste approach where segments from the transcripts were copied and assigned to the generated codes.

\section{Results}

\section{Characteristics of Study Participants}

Between January 2010 and January 2013, there were 1624 infants registered in the EID clinic at Mbarara Regional Referral hospital. One third of these infants (33\%) were excluded from analysis because the mother's ISS clinic identification number to link the infant and mother's data was missing in the EID record. Also, 1.6\% of the remaining 1091 infants, was left out because they were dead or moved to other clinics i.e., they did not contribute towards obtaining the desired outcome. A cohort of 1073 mother-infant pairs enrolled into PMTCT and followed up for 18 months was therefore analysed (Table 1).

\section{Proportion of HIV-exposed infants lost to follow-up}

Out of the total cohort of 1073 infants analysed, 515 (48\%; 95\% Cl: $45 \%$ to $51 \%)$ were LTFU by age 18 months; they did not complete follow-up to the point of being discharged and were not declared deceased. The findings revealed that lost to follow-up was 261 (24\%) at 3 months, 308 (29\%) at 6 months 
while 436 (40.6\%) and 515 (48\%) were lost to follow up at 12 and 18 months into care respectively (Table 2).

\section{Proportion of mother to child transmission (sero-conversions)}

Out of the 515 infants who were LTFU, 7 infants (1.4\%) had tested positive for HIV and 508 had an unknown HIV status (had negative 1st or 2nd PCR results but didn't return for final testing). Of the 558 who were discharged, 20 infants (3.6\%) tested positive for HIV and were referred for care into the ART clinic.

\section{Factors associated with loss to follow up of HIV-exposed infants}

Mother's age, distance from home to hospital and family planning use were found to be associated with loss to follow up following the bivariate analysis conducted. Compared to mothers within the ages 18-23 years, $p=0.002(\mathrm{OR}=0.6 ; 95 \% \mathrm{Cl} 0.44-0.82)$, mothers with age $>30$ years were least likely to have their infants lost to follow up. Again, those with 25-29 years were less likely to have their infants lost to follow up compared to mothers with $18-23$ years of age, $p=0.05(\mathrm{OR}=0.57 ; \mathrm{Cl} 0.42-0.76)$. Long distances from home that took mothers more than 1 hour to get to the hospital were associated with infant LTFU, $p=0.05$ $(\mathrm{OR}=1.31,95 \% \mathrm{Cl} 0.99-1.73)$. Infants whose mothers were not using any family planning method were more likely to be lost to follow up, $p=0.01$ (OR=1.48, $95 \% \mathrm{Cl} 1.09-2.02$ ).

Multivariable analysis indicated that young age of mother; long distance from home to hospital; and failure to use family planning methods were independently associated with loss to follow-up (Table 3). Compared to those infants whose mothers were in the 18-23 years age category, the odds of LTFU were lower among those whose mothers fell in the 24-29 age category. The odds of loss to follow-up were higher among babies whose mothers were reported to come from faraway places to hospital at enrolment in ISS clinic compared to those whose mothers were coming from near.

The odds of loss to follow-up were higher among babies whose mothers reported they did not use family planning compared to those whose mothers were using family planning at enrolment in ISS clinic (Table 3)

\section{Findings from in-depth interviews}

Factors identified from qualitative interviews were grouped facility-level and client barriers.

\section{Facility level factors}

\section{Waiting time}

Mothers pointed out waiting time as one of the reasons why they did not return their babies to the facility for follow-up. One respondent had this to say: 
"The other thing is that we spend a lot of time here. You try to come early so that by midday you are going back home but you find yourself returning home in the evening. When it comes to lunch time, the health workers close and go for lunch, by the time they come back, time has already elapsed and yet you wanted to go look for some money to feed the children," The young mother explained.

Waiting time was also mentioned by one health worker as a hindrance to child follow-up. A nurse who works with the HIV-exposed infants' clinic when quizzed on why mothers were not returning their children for follow-up, submitted that: "It might be the long queue at the clinic especially for working class mothers."

\section{Client based factors:}

\section{Distance to health facility}

Travelling long distances to the health facility was reported as a barrier to adhering to EID clinic schedules. A mother from Bushenyi had this to say:

"I come all the way from Bushenyi with a child. We spend a whole day here with nothing for my child to eat. Sometimes, the health workers are slow and by the time we are out of the queue, the child is already very hungry. So, when I thought about the long journey to and from the health facility and how very challenging it is, I decided to leave my child at home since I was not breastfeeding and the child's first results were negative," she justified.

\section{Short childbirth spacing and multiple visits from single household:}

When children are born too close to each other, this can affect their HIV care because the burden of carrying two children to care is enormous for the mother. This is worsened if mother and children have different follow-up dates.

"Like I said, my second child came a bit too early after the first. So, the first didn't come to the clinic the number of times she was supposed to have come. She came in once. But the second one because she was sick, I had to bring her, and then bring myself, and then bring her... you couldn't ask for the same day, you would get disturbed... being called here and there. So that was challenging. When my daughter was still sick, I had to bring her every month, then bring myself as well. But when she got better, that kind of changed. That's why I didn't even bother bringing her for discharge," the young mother explained.

\section{Discussion}

The outcome of this research reveals that loss to follow-up rates (24\% at 3 months, $29 \%$ at 6 months, $40.6 \%$ at 12 months and $48 \%$ at 18 months) among infants who were enrolled for PMTCT services between January 2010 and January 2013 were progressively high. This finding could be attributed to the existing socio-demographic, socio-economic and behavioural characteristics of mothers. In addition, facility-based factors could also have influenced this high LTFU rate. Compared to that reported in a 
similar cohort in Western Kenya by [9] which reported a rate of $27.4 \%$ by 18 months follow-up, the rate of LTFU in this study cohort is higher. The relatively low MTCT rate in infants who complete follow-up (3.6\%) could be justified by the new option $\mathrm{B}+$ treatment guidelines for prevention of mother-to-child transmission of HIV introduced by the Ministry of Health of Uganda. According to this option, pregnant women who test HIV-positive are immediately placed on life time ART on their first antenatal visit. The constant radio adverts on PMTCT may also have contributed to this reduced transmission rate considering that in the [3] country report, vertical transmission of HIV was reported to account for $20 \%$ of all HIV infections in Uganda. Comparative to rates reported in Tanzania and Ethiopia, the rate of MTCT in this study cohort is low. Mwendo and colleagues [10] reported a 9.6\% MTCT rate in Kilimanjaro region, Tanzania. The variation in rate of transmission between this study and the one carried out in Tanzania could be attributed to accessibility of health services by study participants in both studies. Majority of the mothers in this study were urban dwellers whereas participants in the study in Tanzania were mainly rural residents who were more likely to face difficulty in accessing PMTCT services. While Berhan and colleagues [11] reported $10.1 \%$ HIV prevalence among HIV exposed infants in a facility-based study carried out in Amhara region, Ethiopia, the variation in rate of transmission between these two studies could be attributed to country-level differences in response to elimination of MTCT. Whereas Uganda has a HIV prevalence rate among pregnant women of $6.1 \%$ [12] compared to $1.2 \%$ of Ethiopia, the MTCT rate is lower in Uganda at 20\% [3] compared to $33 \%$ in Ethiopia[11].

These researchers found out that among mother-infant pairs receiving HIV care at Mbarara Regional Referral hospital PMTCT clinic, young maternal age, travelling long distances to the hospital and failure to use family planning were independently associated with loss to follow-up. In Uganda, the median age at first marriage among women within the ages $25-49$ years is 18.2 years. Early marriage and early pregnancy coupled with HIV infection brings a lot of stress to young women who would still be girls and in school under normal circumstances. Although early marriage and pregnancy is a common occurrence in Uganda, there is still a lot of stigma and young women of school going age find it hard to seek family planning services. Young age of the mother has been found to be associated with LTFU in previous studies in Mali and Malawi [13-14]. Whereas parity was not significantly associated with loss-to-followup, qualitative results indicated that number of children a woman has to take for EID services may be an important factor influencing loss-to-follow-up. Failure to use family planning leads to short childbirth spacing and multiple visits from a single household especially if scheduling of clinic visits is not familycentred. Mothers may also find it difficult to carry more than one child to the hospital for every appointment thereby leading to LTFU. Again, walking long distances to access a health facility was significantly associated with LTFU. This was confirmed in the qualitative interviews where mothers revealed that lack of transport and long distances to health facility were barriers to completing follow-up. This may be attributable to unavailability of PMTCT services at lower health centres. Stigma may also be one of the contributing factors to why women travel long distances and seeking PMTCT services.

Qualitative data revealed a complex interplay between facility-level and client-level barriers. For example, the influence of long distance to the health facility on infant follow-up may be compounded by delays at the health facility due to long waiting time and the mother's inability to afford out-of-pocket expenses for 
lunch during clinic visits. In addition, qualitative data suggests that mothers' decisions to take or not to take their infants for EID follow-up visits may be influenced by their own interpretation of initial negative PCR results amidst the search for reasonable manoeuvres to minimise client burden of seeking HIV care.

\section{Limitations}

The researchers used routine data from the ISS clinic database and some information was missing. Thirty three percent of the eligible study participants were dropped due to lack of mothers' clinic identification numbers which made it impossible to find their data in the ISS client database. This in part reflects the poor data recording practices in hospital settings which could affect validity of results from analyses of routine patient care data. If missingness of mother's client ID was in some way related to the LTFU, it means that our results could be an under-estimate of LTFU rate. However, we do not have a reason to assert that this was the case. While it may be reasonable to be cautious with interpretations of our quantitative results, we note that they are well corroborated by qualitative data.

The quantitative analyses utilised variables routinely collected during patient care and did not consider other important factors such as waiting time, behaviour of health workers and information held by mothers regarding care for exposed infants. However, through in-depth interviews, the researchers were able to highlight some of the important variables not found in electronic databases.

Due to lack of enough funds to carry out tracking of lost infants, these researchers only interviewed mothers who responded to phone calls and managed to travel to the clinic for the interviews. The researchers could have missed out on a number of factors affecting mothers in their homes. A thorough investigation of causes of LTFU and sero-conversion rates of HIV exposed infants is warranted to provide stronger evidence for design and funding of effective programs to reduce LTFU.

Despite these limitations however, we firmly assert that the study findings are useful to inform implementation of PMTCT in large ART clinics in Uganda and other comparable settings.

\section{Conclusion}

This research paper reveals a high rate of loss to follow up among HIV-exposed infants enrolled at Mbarara Hospital PMTCT clinic. Young maternal age, long distance to health facility and failure to use family planning were meaningfully associated with LTFU. Short childbirth spacing, child wellbeing, lack of transport, lack of partner support, client waiting time, and limited waiting space at health facility, behaviour of health workers, and bridge of confidentiality may also influence LTFU. In a bid to eliminate mother-to-child HIV transmission and enhance subsequent care and treatment for HIV exposed infants, strategies for retaining children who are at the greatest risk of morbidity and mortality in care need to address issues identified in this study.

Our findings suggest incorporating family planning services in the ART and PMTCT clinics could reduce loss to follow-up of HIV exposed infants. Young mothers should be targeted with information about the 
importance of keeping their babies' clinic appointments even when their babies are not ill in order to reduce children's risk of infection and also provide the necessary care to those already infected.

\section{Recommendation}

Since thirty three percent (33\%) of the eligible study participants were dropped due to lack of mothers' clinic identification numbers which made it impossible to find their data in the ISS client database, we therefore recommend that, at every visit of the mother to the clinic, the health care provider to ensure that identification numbers be given to all pregnant women who come to the hospital for clinic.

\section{Abbreviations}

ANC: Antenatal clinic, LTFU: Loss to follow-up, MTCT: Mother to child HIV-transmission, HIV: Human Immunodeficiency Virus, PMTCT: Prevention of Mother-to- Child HIV-transmission, EID: Early Infant Diagnosis, PCR: Polymerase Chain Reaction, MRRH: Mbarara Regional Referral Hospital

\section{Declarations}

\section{Ethical approval and consent to participate}

Ethical approval was obtained from the Mbarara University of Science and Technology (MUST), Faculty of Medicine Research and Ethics Committee and Institutional Review Committee (MUST IRB). Permission to carry out the study was sought from Mbarara Hospital ISS clinic authorities. The study used hospital records following approval. There was an interview between the parents and guardians of the children and the health workers and informed consents were signed by all participants. In this study, there were no interviews between the children and the researcher. Only hospital records were used in this case.

\section{Consent for publication}

Not applicable

\section{Availability of data and material}

De-identified data that support the findings of this study are available from the first author, Rogers Ankunda but restrictions apply under license for the current study. The data may be made publicly available upon reasonable request and with permission of ISS clinic located in Mbarara Regional Referral Hospital, Mbarara, Uganda.

\section{Competing interests}

The authors declare no competing interest.

\section{Funding}


No funding for this study

\section{Authors' contributions}

RA, SNC, CA, CNN, FWS and TE conceived and designed the study. RA and TE implemented the study and conducted data analysis. TE supervised the study, RA, SNC, CA, KT and TE interpreted study results: RA and CA wrote the first draft of the manuscript. CA, KT, SNC, CNN and FSW reviewed and corrected the draft manuscript. All authors read and approved the final manuscript.

\section{Acknowledgements}

We are grateful to all who participated in this research.

\section{References}

1. HIV/AIDS UNP on. Global Plan towards the Elimination of New HIV Infections among Children by 2015 and Keeping their Mothers Alive. 2011.

2. HIV/AIDS. JUNP on, UNICEF. Children and AIDS: Fifth Stocktaking Report, 2010. 2010. UNICEF.

3. Uganda A. Commission: UNGASS Country Progress Report Uganda: January 2006 to December 2007. Comm UA Gov Uganda. 2008.

4. UNICEF. For every child, end AIDS: seventh stocktaking report, 2016. 2016. UNICEF.

5. Larsson EC, Ekström AM, Pariyo G, Tomson G, Sarowar M, Baluka R, et al. Prevention of mother-tochild transmission of HIV in rural Uganda: Modelling effectiveness and impact of scaling-up PMTCT services. Glob Health Action. 2015;8(1):26308.

6. Kalembo FW, Zgambo M. Loss to followup: a major challenge to successful implementation of prevention of mother-to-child transmission of HIV-1 programs in sub-Saharan Africa. Isrn Aids. 2012;2012.

7. Kaplan R, Orrell C, Zwane E, Bekker L-G, Wood R. Loss to follow-up and mortality amongst pregnant women referred to a community clinic for antiretroviral treatment. AIDS. 2008;22(13):1679.

8. Dean AG, Sullivan KM, Soe MM. OpenEpi: Open source epidemiologic statistics for public health, version. 2014.

9. Nyandiko WM, Otieno-Nyunya B, Musick B, Bucher-Yiannoutsos S, Akhaabi P, Lane K, et al. Outcomes of HIV-exposed children in western Kenya: efficacy of prevention of mother to child transmission in a resource-constrained setting. JAIDS J Acquir Immune Defic Syndr. 2010;54(1):42-50.

10. Mwendo EM, Mtuy TB, Renju J, Rutherford GW, Nondi J, Sichalwe AW, et al. Effectiveness of prevention of mother-to-child HIV transmission programmes in $\mathrm{K}$ ilimanjaro region, northern $\mathrm{T}$ anzania. Trop Med Int Heal. 2014;19(3):267-274.

11. Berhan Z, Abebe F, Gedefaw M, Tesfa M, Assefa M, Tafere Y. Risk of HIV and associated factors among infants born to HIV positive women in Amhara region, Ethiopia: a facility based retrospective study. BMC Res Notes. 2014;7(1):876. 
12. UNICEF. State of the world's children: Celebrating 20 years of the convention on the rights of the child. 2009. Unicef.

13. Organization WH. A report on the misdiagnosis of HIV status. 2014.

14. Mute T, Akondé A, Doumbia A. The prevention of mother-to-child transmission of HIV-1 in Mali HIV-1positive pregnant women and loss to follow-up in the Segou region. In: 6th IAS Conference on HIV Pathogenesis and Treatment Abstract no. MOPE480. 2011.

\section{Tables}

Table 1: Characteristics of Study Participants

Table 2: Proportion of loss to follow up of infants against time

\begin{tabular}{|lccc|}
\hline Time of LTFU & Cumulative LTFU n (\%) & Active n (\%) & 95\% CI \\
\hline At 3 months & $261(24.3)$ & $812(75.7)$ & $0.217-0.269$ \\
\hline At 6 months & $308(28.7)$ & $765(71.3)$ & $0.260-0.314$ \\
& & & \\
\hline At 12 months & $436(40.6)$ & $637(59.4)$ & $0.376-0.436$ \\
\hline At 18 months & $515(48.0)$ & $558(52.0)$ & $0.450-0.510$ \\
\hline
\end{tabular}

Table 3: Bivariate and Multivariate analysis of factors associated with loss to follow up of HIV exposed infants 


\begin{tabular}{|c|c|c|c|}
\hline Characteristics & $\begin{array}{c}\text { Total Cohort } \\
(n=1073)\end{array}$ & $\begin{array}{c}\text { LTFU } \\
(n=515)\end{array}$ & $\begin{array}{c}\text { Discharged } \\
(n=558)\end{array}$ \\
\hline Age (median IQR) & $26(23-30)$ & $26(22-30)$ & $27(24-30)$ \\
\hline \multicolumn{4}{|l|}{ Religion: n (\%) } \\
\hline Catholic & $266(24.8)$ & 133(25.8) & $133(23.8)$ \\
\hline Protestant & $465(43.3)$ & $224(43.5)$ & $241(43.2)$ \\
\hline Muslim & 107(9.9) & $47(9.1)$ & $60(10.7)$ \\
\hline Other & $40(3.7)$ & $24(4.6)$ & 16(3.1) \\
\hline Missing & $195(18.2)$ & $87(16.9)$ & $108(19.4)$ \\
\hline \multicolumn{4}{|l|}{ Marital Status: n (\%) } \\
\hline Single & $81(7.5)$ & $39(7.5)$ & $42(7.5)$ \\
\hline Married & $706(65.8)$ & $348(67.6)$ & $358(64.2)$ \\
\hline Separated & $115(10.7)$ & $52(10.1)$ & $63(11.3)$ \\
\hline Divorced & $53(4.9)$ & $24(4.7)$ & $29(5.2)$ \\
\hline Widowed & $71(6.6)$ & $32(6.2)$ & $39(6.9)$ \\
\hline Missing & $47(4.4)$ & $21(4.1)$ & $26(4.7)$ \\
\hline \multicolumn{4}{|l|}{ Education: $\mathrm{n}(\%)$} \\
\hline None & $30(2.8)$ & $16(3.1)$ & $14(2.5)$ \\
\hline Primary & $533(49.7)$ & $269(52.2)$ & $264(47.3)$ \\
\hline Secondary & $224(20.9)$ & $111(21.5)$ & $113(20.3)$ \\
\hline Tertiary & $62(5.8)$ & $23(4.5)$ & $39(6.9)$ \\
\hline Missing & $224(20.9)$ & $97(18.9)$ & $127(22.8)$ \\
\hline Parity (Median IQR) & $2(1-4)$ & $2(1-4)$ & $2(1-4)$ \\
\hline \multicolumn{4}{|l|}{ Income: $\mathrm{n}(\%)$} \\
\hline$<100,000$ & $622(57.9)$ & $302(58.6)$ & $320(57.3)$ \\
\hline $100,001-250,000$ & $101(9.4)$ & $46(8.9)$ & $55(9.8)$ \\
\hline $250,001-500,000$ & 18(1.7) & $9(1.7)$ & $9(1.6)$ \\
\hline$>500,000$ & $10(0.9)$ & $4(0.8)$ & $6(1.1)$ \\
\hline Missing & $322(30.0)$ & $154(29.9)$ & $168(30.1)$ \\
\hline \multicolumn{4}{|l|}{ Distance : $\mathrm{n}(\%)$} \\
\hline Short & $529(49.3)$ & $243(47.2)$ & $286(51.3)$ \\
\hline Long & $315(29.4)$ & $165(32.0)$ & $150(26.9)$ \\
\hline Missing & $229(21.3)$ & $108(20.9)$ & $121(21.7)$ \\
\hline \multicolumn{4}{|l|}{ Occupation: $\mathrm{n}(\%)$} \\
\hline Unemployed & $227(21.2)$ & $120(23.3)$ & $107(19.2)$ \\
\hline Student & $18(1.7)$ & $8(1.5)$ & $10(1.8)$ \\
\hline Civil Servant & $29(2.7)$ & $13(2.5)$ & $16(2.9)$ \\
\hline Business & $209(19.5)$ & $93(18.1)$ & $116(20.8)$ \\
\hline Armed forces & $2(0.2)$ & $1(0.2)$ & $1(0.2)$ \\
\hline Farmer & $316(29.4)$ & $160(31.1)$ & $156(27.9)$ \\
\hline Other & $152(14.2)$ & $67(13.0)$ & $85(15.2)$ \\
\hline Missing & $120(11.2)$ & $53(10.3)$ & $67(12.0)$ \\
\hline \multicolumn{4}{|c|}{ Alcohol Consumption: $\mathbf{n}(\%)$} \\
\hline No & $706(65.8)$ & $338(65.6)$ & $368(65.9)$ \\
\hline Yes & $170(15.8)$ & $84(16.3)$ & $86(15.4)$ \\
\hline Missing & $197(18.4)$ & $93(18.1)$ & 104(18.6) \\
\hline
\end{tabular}




\begin{tabular}{|lccc|} 
No & $70(6.5)$ & $33(6.4)$ & $37(6.6)$ \\
Yes & $772(71.9)$ & $373(72.4)$ & $399(71.5)$ \\
Missing & $231(21.5)$ & $109(21.2)$ & $122(21.9)$ \\
\hline Family planning use: & & \\
No & $200(18.6)$ & $112(21.8)$ & $88(15.8)$ \\
Yes & $873(81.4)$ & $403(78.2)$ & $470(84.2)$ \\
\hline
\end{tabular}




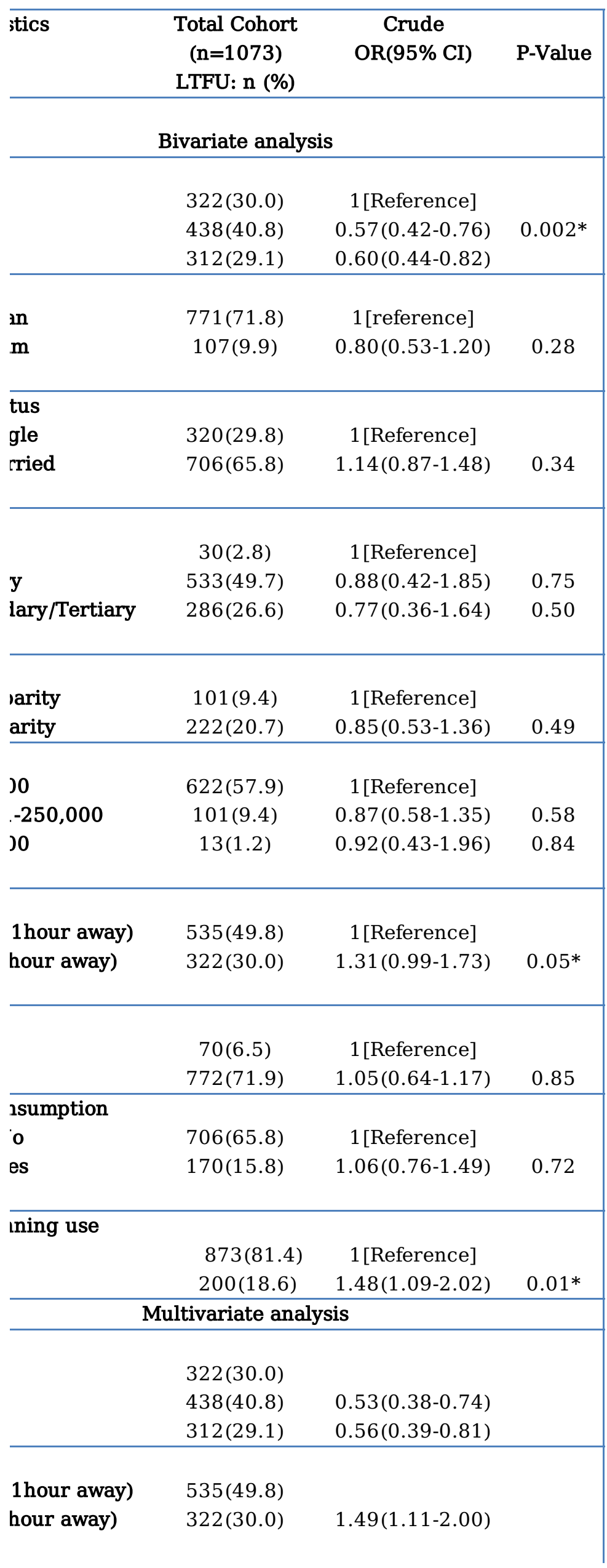


Ining use

3

ว
$873(81.4)$

$200(18.6) \quad 1.83(1.29-2.60)$

*Statistically significant, CI= Confidence Interval 